\title{
Open to change
}

The 'new normal' way of life for coping with the COVID-19 outbreak is a work in progress. When we move on, we should keep some of our adaptations rather than return to the old ways, for a more open and equitable way of working.

B y all accounts, 2020 has been A Bad Year. Even without the COVID-19 pandemic, we've witnessed countless extremes in natural disasters - literally, the naming of Atlantic storms is deeper into the Greek alphabet than ever before. Anxiety levels are running at an all-time high thanks to the 24-hour (bad) news cycle, with the latest blow to astronomy being the demise of the Arecibo Observatory. And yet there are glimmers of hope as the year winds down. In particular, COVID-19 vaccines that are claimed to be over $90 \%$ effective are on the horizon, and have us all dreaming of a return to normal within six months.

But do we actually want the old normal back? For many activities, such as seeing friends and family, travelling or having access to medical professionals for non-urgent health conditions: yes, absolutely. For others, however, our forced adaptation to keep businesses operating during various levels of restrictions and lockdowns has helped us evolve. Going back to our old habits would be a lost opportunity to address entrenched inequalities. Take commuting to work, for instance. The new normal of working from home (for those fortunate enough to do so) has demonstrated that office jobs need not be full-time in the office, each and every day. Hence, the new, new normal should adopt a flexible approach where people can choose how many days they would like to work from home, and during which hours. Besides reducing commuter stress and environmental impact, flexible working helps parents and carers, who might otherwise not be able to juggle rigid working hours with caring duties. Quite simply, maintaining flexible working would increase the level of diversity within the workforce and improve their quality of life.

\section{"Going back to our old habits would be a lost opportunity to address entrenched inequalities."}

A step beyond commuting is attending conferences. The switch to virtual conferences in 2020 has had the added benefit of making them more inclusive. Whether due to financial, physical or scheduling constraints, many students and academics are unable to travel to conferences, thus missing out on networking, giving talks or presenting posters. Going online is more affordable not to mention, lower in carbon emissions - but it isn't quite the same experience; staying engaged for hours at a time can be a challenge while staring passively at a screen, especially when the talks are taking place several time zones away and the speakers freeze periodically. Moreover, despite Zoom rooms and Slack channels, we haven't yet found the digital equivalent of a spontaneous conversation in the long coffee queue. Coming out of this pandemic, a return to old-style conferences will be welcome, but organizers should offer the option for remote participation on an equal footing with in-person attendance.

Another lesson from COVID-19 is that cooperation is essential for tackling a planet-scale crisis, as recognized in SDG-17 of the United Nations Sustainable Development Goals. The sharing of data, information and research across the globe led to rapid advances in treatment and vaccine technologies. For Nature and its sister journals, all published articles on COVID-19 research are freely accessible while the outbreak remains a public health emergency. The general Coronavirus Collection covers research into the detection, treatment and evolution of the infection, the epidemiology of viral diseases and their wider societal effects. For example, the Collection includes a Correspondence from this issue of Nature Astronomy that raises awareness of the detrimental effects of lockdown on female Italian researchers in astronomy and astrophysics.

Open research is a cornerstone of Nature Research and we encourage authors to post preprints on preprint servers and to self-archive the final submitted version of their manuscripts six months after publication. Each of our authors also gets a link to a free, sharable PDF version of the published paper through our SharedIt initiative. These links can be emailed, shared on social media, posted on websites and so forth. We also encourage the sharing of data, codes and methodologies to maximize transparency and reproducibility.

"Open research is a cornerstone of Nature Research and we encourage authors to post preprints on preprint servers and to self-archive the final submitted version of their manuscripts."

The next step in increasing dissemination is open access publishing. From January 2021, Nature and the Nature Research journals will be offering authors the opportunity to publish their work under an open access policy, so that anyone in the world can access their paper and associated materials, immediately and without charge. In this case, the cost of publishing is shouldered by the authors' funders. A growing number of funding bodies and institutions are stipulating open access, and this new publishing option will support researchers in meeting such requirements. In addition, some authors may wish to choose this option simply because they consider it the best way to share their research.

Our standard method of publishing papers, where the publishing costs are not incurred by the authors, but spread between many readers through a subscription model, will continue to be available in the normal way. By offering both publishing options, we hope that the best research, vetted by two to three (and sometimes more) qualified reviewers, will reach the widest possible audience. We will be open to all authors.

Published online: 8 December 2020 https://doi.org/10.1038/s41550-020-01281-0 